\title{
The quality-of-life impact of prostate cancer treatments.
}

\author{
Jaspreet Singh
}

Thomas Jefferson University

Edouard J. Trabulsi

Thomas Jefferson University

Leonard G. Gomella

Thomas Jefferson University

Follow this and additional works at: https://jdc.jefferson.edu/urologyfp

Part of the Urology Commons

Let us know how access to this document benefits you

\section{Recommended Citation}

Singh, Jaspreet; Trabulsi, Edouard J.; and Gomella, Leonard G., "The quality-of-life impact of prostate cancer treatments." (2010). Department of Urology Faculty Papers. Paper 7.

https://jdc.jefferson.edu/urologyfp/7

This Article is brought to you for free and open access by the Jefferson Digital Commons. The Jefferson Digital Commons is a service of Thomas Jefferson University's Center for Teaching and Learning (CTL). The Commons is a showcase for Jefferson books and journals, peer-reviewed scholarly publications, unique historical collections from the University archives, and teaching tools. The Jefferson Digital Commons allows researchers and interested readers anywhere in the world to learn about and keep up to date with Jefferson scholarship. This article has been accepted for inclusion in Department of Urology Faculty Papers by an authorized administrator of the Jefferson Digital Commons. For more information, please contact: JeffersonDigitalCommons@jefferson.edu. 


\section{The Quality-of-Life Impact of}

\section{Prostate Cancer Treatments}

Jaspreet Singh, Edouard J. Trabulsi,

Leonard G. Gomella

Department of Urology

Kimmel Cancer Center

Thomas Jefferson University

Philadelphia, PA

Correspondence:

Leonard G. Gomella, MD

Chairman, Department of Urology

Kimmel Cancer Center

Thomas Jefferson University, Philadelphia, PA

1025 Walnut Street, Suite 1112

Philadelphia, PA 19107

Office (215) 955-6961

Fax (215) 923-1884

Leonard.gomella@jefferson.edu

Version 6/27/2010

For Current Urology Reports 


\begin{abstract}
Many options exist for the treatment of localized prostate cancer. In the decision to choose a therapeutic option for localized disease, many variables need to be considered such as tumor characteristics, clinical stage, the patient's overall health and life expectancy, and preferences of both the physician and patient. Another important consideration is the healthrelated quality of life (HRQOL) implications of a given treatment option. The importance of HRQOL relative to the potential side effects of prostate cancer treatments has grown over the past few years. Although our collective awareness has increased, objective data on HRQOL for prostate cancer treatment are lacking due to a paucity of prospective clinical trial data. This review defines the concept of HRQOL, discusses what is currently known about the impact of various treatments on HRQOL, and summarizes the recent literature in this area relating to the management of localized prostate cancer.
\end{abstract}

Keywords: prostate cancer, radical prostatectomy, external beam radiation therapy, brachytherapy, active surveillance, health related quality of life, complications 
Introduction

Prostate-specific antigen (PSA)-based screening for prostate cancer $(\mathrm{CaP})$ has resulted in a stage migration, leading patients to be diagnosed earlier in the disease process, at a younger age and with lower risk factors $[1,2]$. Data from the large Cancer of the Prostate Strategic Urologic Research Endeavor (CaPSURE) database demonstrated a significant increase in the proportion of patients with low-risk tumor characteristics from $29.8 \%$ in $1989-1992$ to $45.3 \%$ in 1999-2001 [2]. More importantly, the robust favorable long-term survival in low-risk CaP has become even more pronounced in recent years because PSA testing has led to cancer diagnosis at an earlier point of the disease course and to be alive longer with their disease. In fact, progression-free survival following local intervention for low-risk CaP approaches $95 \%$ at 10 years [3].

Various established treatment options exist for clinically localized $\mathrm{CaP}$, such as active surveillance (AS), radical prostatectomy (RP), radiotherapy (RT), and brachytherapy (BT). Some of these, such as radiation therapy, can include hormonal therapy in the regimen. Unfortunately, there have been no adequately sized randomized clinical trials to directly compare the various interventions for $\mathrm{CaP}$, and past attempts to compare interventions such as brachytherapy and radical prostatectomy have not been successful. Therefore, in the setting in which one treatment option cannot be ruled superior to another, the side effect profiles of the different treatment options are often considered with making a treatment decision. Understanding the health related quality of life (HRQOL) enables the urologist and individual patient to have realistic expectations and to optimize treatment decisions for the $\mathrm{CaP}$ patient. Customizing risk profile to each patient can also prove to be important, such as taking into consideration the patient's baseline health, anxiety, and expectations. For example, a patient who has a history of 
obstructive urinary symptoms related to symptomatic benign prostatic hyperplasia (BPH) may benefit from surgical treatment of his cancer, which would also alleviate his symptoms of BPH. A summary of common interventions for $\mathrm{CaP}$ and associated HRQOL side effects is listed in Table 1. Because there is limited evidence that any of the treatments for localized disease is superior to another in terms of overall survival, patients must consider other variables when choosing therapy. One of these other considerations is quality of life (QOL). Recognition of the importance of HRQOL related to the side effects of $\mathrm{CaP}$ treatments has grown over the years. Although the awareness by physicians and patients has increased, there is still a paucity of objective data of HRQOL for CaP treatment, primarily due to the relative lack of prospective trials. It is critically important that clinicians understand the impact of $\mathrm{CaP}$ and its effect on HRQOL so that they can better counsel their patients and make early interventions that improve patient outcomes. The aim of this review is to briefly establish the definition of HRQOL as well as the known consequences of $\mathrm{CaP}$ treatments on HRQOL, and review updates on this topic in recent literature concerning the management of localized $\mathrm{CaP}$.

\section{Definition and Measurement of HRQOL in $\mathrm{CaP}$}

HRQOL is measured using standardized questionnaires, which collect patient-centric data and provide an objective assessment and perception of both generic and disease specific domains. In the past, health care providers would document what was perceived QOL, but a more accurate measure includes patient-driven perception of personal health, the ability to function in life, and overall sense of satisfaction with life experiences [4]. The ultimate goal of HRQOL is to detect variation in the natural history of disease and treatment effects not described by vital status or observations recorded in the clinical record [5]. There are several validated 
questionnaires, also commonly described as "instruments" or "tools," developed for patients with $\mathrm{CaP}$.

The University of California Los Angeles Prostate Cancer Index (UCLA-PCI) was the first validated questionnaire specifically designed to measure HRQOL in localized CaP patients. General HRQOL domains were borrowed from the established Medical Outcomes Study Short Form (SF-36), and prostate-specific domains were included: urinary function and bother, sexual function and bother, and bowel function and bother. Briefly, the SF-36, which is perhaps the most widely used tool for general HRQOL, consists of eight scaled scores: vitality, physical functioning, bodily pain, general health perceptions, physical role functioning, emotional role functioning, social role functioning, and mental health [5].

The Expanded Prostate Cancer Index Composite (EPIC) is a comprehensive instrument designed to evaluate patient function and bother after $\mathrm{CaP}$ treatment. Content from the original UCLA-PCI was expanded with guidance from a cohort of localized CaP patients and an expert panel comprised of urologic oncologists, radiation oncologists, survey researchers, and CaP nurses. This extensive panel found a need to augment the UCLA-PCI with items to capture additional concerns relevant to BT, RT, RP, and androgen deprivation. Symptom-specific bother items corresponding to each symptom item were added to elicit multi-item bother scales for each HRQOL domain [4].

The European Organization for Research and Treatment of Cancer Core Quality of Life Questionnaire (EORTC QLQ-C30) is a 30-item self-reported questionnaire developed to assess the QOL of cancer patients. It is grouped into five functional subscales: role, physical, cognitive, emotional, and social functioning. In addition, there are three multi-item symptom scales (fatigue, pain, and nausea and vomiting), individual questions concerning common symptoms in 
cancer patients, and two questions assessing overall QOL. The EORTC QLQPR25 was

developed to incorporate an additional 25-item subset specific to $\mathrm{CaP}$ [6].

\section{Effect of Patient Treatment Options on Patient QOL}

\section{Active Surveillance}

$\mathrm{CaP}$ can progress slowly and, with or without treatment, a large number of men can live many years after diagnosis. The realization that low-risk $\mathrm{CaP}$ may sometimes be overtreated by surgery or radiation has been an impetus in the development of an active surveillance (AS) protocol. Therefore, men with favorable disease-specific features are candidates for AS, and perhaps can benefit from the avoidance of various side effects of treatment of localized $\mathrm{CaP}$ such as incontinence, bleeding, gastrointestinal toxicity, and erectile dysfunction. Although physical side effects may be avoided with AS, there is an emerging concern that patients may be at an increased risk of psychologic distress. HRQOL was evaluated in 129 men that were enrolled in the Prostate Cancer Research International: Active Surveillance (PRIAS) study. Although for the most part, AS was well tolerated among the cohort, certain risk factors for patients who may not do well on AS emerged. A perception of the physician contributing to the majority of the decision making, a poor physical health score, a high neuroticism (anxiety) score, and a high PSA value all had significant positive associations with lower HRQOL scores [7]. Burnet et al. [8] also noted that AS, as a treatment entity, did not offer higher overall psychological distress compared with other $\mathrm{CaP}$ treatments, but analyses indicated that higher anxiety scores were significantly associated with younger age and a longer time interval from diagnosis. In addition, higher depression scores were significantly associated with a longer interval since diagnosis $(\mathrm{P}<0.05)$, but not with management by AS. Therefore, although AS has 
substantial benefits, it can also have significant effects on QOL, and additional research is needed to define how to minimize the specific QOL domains affected.

The National Cancer Institute (NCI)-endorsed phase III Study of Active Surveillance Therapy Against Radical Treatment in Patients Diagnosed With Favorable-Risk Prostate Cancer (START) trial, currently enrolling patients in the United States and Canada, will compare the disease-specific survival of patients who have favorable risk CaP treated with RP or radical RT at the initial diagnosis with that of patients whose treatment is AS and selective active treatment intervention (either radiation or surgery). In addition to cancer-related outcomes, the study will use a number of QOL instruments to further examine the QOL problems these different approaches may involve [9].

\section{Radical Prostatectomy}

Several trials have shown that RP has significant negative effect on multiple QOL domains such as lower sexual function score, lower urinary function and incontinence scores, and lower physical HRQOL $[10,11 \bullet \bullet, 12],[13 \bullet \bullet]$. Although certain advances have been made that help diminish these side effects, such as nerve sparing prostatectomy (NSP) or roboticassisted radical prostatectomy (RARP), their impact on HRQOL still remains controversial.

Significant controversy exists regarding the outcomes of RARP and open RP. Although there are limited published data on HRQOL outcomes specifically to RARP, one can look at functional outcomes and deduce the effects on QOL from the open RRP patients. Coelho et al. [14] concluded, after evaluating current RARP outcomes, that this technique offers comparable functional outcomes to open RRP. Therefore, RARP should theoretically offer similar HRQOL scores to open RRP. These outcomes are different than those reported in a recent article by Hu et al. [15•], which examined minimally invasive RP (all laparoscopic techniques) and traditional 
open RP. Measured outcomes of incontinence and erectile dysfunction were higher in the minimally invasive group compared with the open RP group, using a dataset of nearly 2000 Medicare patients from the years 2003 to 2007. Among the criticism of the report, the study failed to stratify the patients between RARP and laparoscopic RP, for which a more pronounced learning curve exists. In addition, the time period (2003-2007) that was examined was when minimally invasive prostatectomy was first becoming popular and more widely available in United States, putatively during most surgeons' initial learning curve. Finally, the study did not control hospital and surgeon case volumes, which is known to correlate with outcomes. Nevertheless, the data suggested worse functional outcomes in patients undergoing minimally invasive RP when compared with open RP.

Preserving the neurovascular bundles (NSP) reduces the incidence of impotence post prostatectomy in both open RP and robotic-assisted RP [11••, 16]. In addition, some studies have also suggested that NSP can help improve urinary function. Abel et al. [16] evaluated 91 consecutive patients divided into two cohorts according to the percentage of nerve sparing performed, $>50 \%$ versus $\leq 50 \%$, at the time of RP. Nerve sparing was associated with better urinary function $(\mathrm{P}=0.014)$ and urinary bother $(\mathrm{P}=0.043)$. After adjusting for preoperative variables, the increased NSP group scored 8.4 points higher for urinary function and 8.5 points higher for urinary bother compared with the non- NSP group. Long-term ( $>5$ years) improvement in urinary incontinence was seen in the NSP group by Nandipati et al. [17]. Urinary continence status was defined as completely pad-free or wearing a single pad for protection (dry pad). Overall incontinence rate was reported to be higher in the non-NS than the bilateral NS (29.5\% vs 9.1\%, respectively; $\mathrm{P}<0.05)$. Return of incontinence was also significantly quicker in the bilateral NS group. Although several anecdotal opinions exist, such 
as a more meticulous apical dissection at the time of NSP and more careful overall technique, the exact causal mechanism of NSP in improving urinary function is not known.

\section{Radiotherapy of the Prostate}

Patients undergoing RT for localized CaP can have urinary, sexual, and gastrointestinal tract dysfunction after treatment. However, the symptomatology of urinary dysfunction after RT is different than that of patients who undergo surgery. Unlike surgery, which can result in stress urinary incontinence, RT can result in irritative voiding symptoms, such as urgency, frequency, and urge incontinence, that negatively affect overall urinary function and HRQOL. Also, specific to RT is a higher incidence bowel irritation. BT and RT were both associated with a reduced QOL related to bowel function early after treatment, continuing in some patients for more than a year. Sanda et al. [11••] reported rectal urgency, frequency, pain, fecal incontinence, or hematochezia-caused distress related to bowel function in $9 \%$ of patients a year after RT. The most predominant severe acute toxicity after BT is urinary retention requiring catheterization [18]. In fact, Roeloffzen et al. [18] reported that acute urinary retention after prostate BT has a significant negative impact on patients' HRQOL up to 6 years after treatment, in terms of both global QOL measures and urinary symptom scores. Proper selection of patients can minimize the risk of retention following BT.

Postoperative $\mathrm{RT}$ for $\mathrm{CaP}$ in a salvage or an adjuvant setting presents a unique challenge, in that maximizing urinary continence and potency prior to delivery of RT is of concern. Pinkawa et al. [19] evaluated 101 patients prospectively that received RT for positive surgical margins and/or persisting or rising PSA levels after RP. Median time between RP and beginning of RT was 16 months. Function and bother scores at baseline and different time intervals were 
recorded after RT. The time between RP and RT did not correlate with a significant difference in function scores. RT-associated sexual toxicity played a minor role in this study because only $7 \%$ of patients reported erections firm enough for intercourse. HRQOL scores in the urinary and bowel domains decreased significantly in the acute phase. Urinary scores recovered rapidly within the first weeks after RT. On the other hand, bowel scores remained significantly lower for a longer period of time, and only recovered back to baseline 1 year after RT. Twenty-nine patients received a course of androgen deprivation for a median duration of 9 months prior to RT. HRQOL scores in this subgroup decreased in the hormonal domain but returned back to baseline 1 year after RT.

Androgen deprivation therapy is well known to have a significant impact on hormonal HRQOL domain. Symptoms in this category include hot flashes, gynecomastia, lack of energy, weight gain, bone loss, increased risk of metabolic syndrome, and depressive mood changes. These concerns are increasingly relevant in the measurement of consequences from localized $\mathrm{CaP}$ treatment because the use of neoadjuvant and adjuvant hormonal therapy has become more common with RT. Neoadjuvant hormonal therapy can also be used for cytoreduction for patients with large prostates, to make them eligible for BT or surgical intervention. Side effects of androgen deprivation in localized disease must be discussed with patients, as their administration can have a significant and long-lasting impact on the QOL of patients.

\section{Physician Awareness of HRQOL}

Increased awareness of the impact of cancer on HRQOL of patients and accurate study of HRQOL have been slow to permeate the field of urologic oncology. A study by Sonn et al. [20•] examined the frequency of various impairment factors, such fatigue, pain, and sexual, urinary, 
and bowel dysfunction who had undergone RP, BT, or External beam radiation therapy recorded by the physician and those self-reported by the patient. The authors compared a modern cohort of a period from 2000 to 2007 with that from 1995 to 2000. Participants independently completed the SF-36 and the UCLA-PCI. Urologists noted impairment in urinary and sexual function more accurately than fatigue or pain. Disagreement between physician and participant ratings did not vary dramatically from short-term to long-term follow-up, or from the earlier to the later cohort, indicating, despite the increased awareness in literature, a need for further provider awareness on QOL issues. As the time from primary treatment increased, patients did report less impairment in all domains, but physicians recorded more improvement than did their patients. This study clearly shows that the wide gap continues to exist between the HRQOL that patients report and that which their physicians assess.

\section{Impact of Screening and Diagnosis on HRQOL}

PSA screening and monitoring can adversely affect patients' anxiety levels and therefore have a diminutive effect on their QOL. Even prior to being diagnosed with $\mathrm{CaP}$, there can be mental anguish and anxiety resulting in a decreased QOL from CaP screening. Carlsson et al. [21] evaluated the anxiety levels of nearly 1800 men with PSA $\geq 3 \mathrm{ng} / \mathrm{mL}$ partaking in the European Randomized Study of Screening for Prostate Cancer. The study illustrated that most men have low to moderate anxiety while waiting for further clinical examination for an elevated PSA, and that $<5 \%$ of men reported high levels of anxiety. Despite the high sensitivity of PSA, variable factors can falsely elevate PSA, including benign enlargement of the prostate. Katz et al. [22] evaluated the effect on psychological well-being of 109 men with an abnormal PSA test or an abnormal digital rectal examination (DRE) but a negative biopsy result. Men with abnormal 
$\mathrm{CaP}$ screening tests report increased cancer related worry and more problems with sexual function, despite having a negative biopsy result. Therefore, false positive results may contribute to a significant decrease in QOL, stressing the point that effective counseling is recommended before $\mathrm{CaP}$ screening and during follow-up.

Increased anxiety is common after being diagnosed with CaP. Understanding the specific domains of well-being affected by the diagnosis can help urologists mediate the negative effects [5]. Love et al. [23] conducted a prospective QOL evaluation of 367 men newly diagnosed with either early or advanced CaP compared with a CaP-free, age-matched comparison group of men. The diagnosis of $\mathrm{CaP}$ had a significant, negative effect on vitality, social functioning, role emotional, and mental status, as measured by the SF-36. Additionally, a greater rate of anxiety was seen in men diagnosed with early-stage (5.2\%) and advanced-stage (5.8\%) CaP compared with aged-matched controls. Strategies that may introduce early recognition and intervention may perhaps slow the deterioration or even improve the patient's QOL, while the patient is going through a treatment process. The elderly population in the United States is no exception. A longitudinal population based study by Reeve et al. [24] examined the impact a diagnosis of $\mathrm{CaP}$ has on HRQOL in the aging population. Patients that were newly diagnosed with prostate cancer experienced statistical declines in physical health (mean score decline of 3.4) and significant decreases in mental health (mean score decline of 2.8) relative to the mean change of the control subjects.

This also illustrates the importance for urologists to understand the impact of $\mathrm{CaP}$ and its treatment on QOL so that patients can be better counseled on expectations and that care providers can provide early interventions for certain QOL domains. 


\section{Preoperative Tumor Characteristics Effect on HRQOL}

Are there certain tumor parameters that help predict which patients with $\mathrm{CaP}$ will do better than their counterparts? Gacci et al. [25] retrospectively reviewed 595 patients who had undergone either RP or RT. Significantly better urinary function and bother scores were noted in patients with localized, low-risk CaP (cT1-2, Gleason score $\leq 3+3$, and PSA $\leq 10 \mathrm{ng} / \mathrm{mL}$ ). Moreover, men with low clinical stage, low Gleason score, and low preoperative PSA had significantly better sexual function scores. Interestingly, there was not a statistical difference in sexual bother scores in the higher risk patients, perhaps reflecting better preoperative counseling and posttreatment expectations of sexual recovery. Moreover, biochemical recurrence also influenced urinary function and bother, as well as sexual function and bother, perhaps reflective of a general decrease in QOL after discovery of cancer recurrence.

\section{HRQOL and Survival}

It has been shown that a higher HRQOL, after controlling from clinical and demographic variables, can improve survival in several types of malignancies, including breast cancer [26]. Aside from a better QOL, is there a similar impact in survival in patients diagnosed with $\mathrm{CaP}$ by merely improving QOL? Sadetsky et al. [27] examined this issue in a recent article, in which they evaluated patients from the CaPSURE database. HRQOL and survival in patients with $\mathrm{CaP}$ were evaluated after controlling for age at diagnosis, type of treatment received, clinical risk classification, and number of comorbidities. Higher levels of physical function and general health were significantly associated with better survival (HR, $0.49 ; 95 \% \mathrm{CI}, 0.32-0.78$, and HR $0.51,95 \% \mathrm{CI}, 0.35-0.75$, respectively). It is not clear if there is a direct causal relationship between HRQOL and survival from CaP, but perhaps this association could provide the stimulus 
to investigate and provide a link between a patient's perception and the severity of the underlying disease.

\section{Spousal Effect on HQROL}

Patients with CaP often rely exclusively on their spouses for support, and therefore, the role as a primary caregiver can be stressful for spouses. Despite the high prevalence of prostate cancer, little information is available on the QOL of men and their spouses during the phases of diagnosis and treatment of CaP. Northouse et al. [28] evaluated patients with $\mathrm{CaP}$ and their spouses through a comprehensive psychosocial assessment across three phases of illness: new diagnosis, biochemical recurrence, and advanced CaP. Spouses' risk for distress scores did not differ from patients' scores, indicating that spouses are not immune to the effects of illness. In fact, spouses had a greater need for the intervention than patients.

Decreases in the QOL from CaP treatment can extend to a patient's spouse or partner. This was shown by Sanda et al. [11••], in which significant distress in the spouse was reported 1 year after initial treatment. As one would expect, the spousal group with the highest erectile dysfunction related distress was reported by $44 \%$ of partners in the RP group, $22 \%$ in the RT group, and only $13 \%$ in the BT group. Obviously, it must be noted that the RP patient group had higher baseline sexual scores and experienced the greatest decrease in QOL score, which is reflected in the spouse group. In addition, the RP group was younger and with less comorbidities and perhaps enjoying a more meaningful sexual life, which is precipitously affected immediately after surgery. Patients' reported bowel and urinary symptoms associated with spousal distress were lower and ranged from $3 \%$ to $7 \%$ in all three groups. Finally, the level of spousal distress arising from a patient's sexual and urinary symptoms after primary CaP treatment was also 
associated with the partner's level of satisfaction with the treatment outcome in multivariate analysis.

Clearly, a health care provider should also include the patient's partner when deciding the appropriate intervention for CaP. Similar to other studies, Badr and Taylor [29] reported that within couples, patients' and their partners' sexual function and satisfaction were very similar. When patients had poor erectile function, their partners were more likely to report that the couple avoided open spousal discussions; this in turn was associated with partners' marital distress. Patients and partners who reported high levels of mutual constructive communication also reported greater marital adjustment, regardless of their own sexual satisfaction. The authors of the study concluded that psychosocial interventions that facilitate healthy spousal communication and address the sexual rehabilitation needs of patients and their partners after $\mathrm{CaP}$ treatment is important.

Several studies have demonstrated that early intervention by the health care provider can improve QOL. Although no difference was seen between patient groups on prostate specific symptoms, spouses in the intervention group reported higher QOL, more self-efficacy, better communication, and less negative appraisal of caregiving, uncertainty, hopelessness, and symptom distress lasting up to 12 months compared with controls [30].

Harden et al. [31] applied the Family Involvement, Optimistic Outlook, Coping Effectiveness, Uncertainty Reduction, and Symptom Management (FOCUS) program, which was originally developed for women with advanced breast cancer and their family caregivers, to prostate cancer patients and partners. Couples identified this as an opportunity to raise difficult issues they might not have discussed with their care provider, and reported high satisfaction with the program. Spouse participation in a supportive, educative intervention increased 
communication with their husbands, lessened their uncertainty, and reduced their negative appraisal of caregiving. Unique to $\mathrm{CaP}$ patients is that the vast majority of partners are women (99\% in this study). Partners in this study reported more satisfaction overall than caregivers of breast cancer patients (64\% were husbands). These differences may be related to the more active caregiving or illness management role that female spouses versus the supportive role caregivers (primarily husbands) of breast cancer patients assume.

\section{HRQOL in Minority Populations}

Controlling for variables, does the black population experience similar QOL compared with the white population undergoing CaP treatment? Jayadevappa et al. [32] conducted a prospective study evaluating QOL and satisfaction with care after RP or RT. Compared with white patients, black patients were less likely to choose RP as primary treatment, although when statistically controlled, ethnicity was not associated with treatment type in statistical significance. Baseline prostate QOL scores were similar in both groups, except the black group reported higher pains in back, legs, or hips. There were a higher percentage of black men accrued from the Veterans Administration (68\% vs $42 \%)$. It is unclear that this difference serves as a discrepancy in the consistency of are delivered between the two groups. The majority of white men were college educated, married, and had an annual income of $>\$ 40,000$ (63\% vs $22 \%$ in the black population). Outcomes of this study included the following: black men took a longer time to return to their baseline generic and sexual bother HRQOL scores; and black men reported poorer outcomes by 12 months for some generic and prostate-specific HRQOL domains, including lower 12- month scores on role physical, role emotional, bodily pain, urinary function, and urinary bother. The etiologies of these discrepancies are unknown, but perhaps it is because 
black patients express lower physician trust than their white counterparts [33]. Alternatively, black patients may have lower satisfaction rates with the services they receive. Regardless, this highlights the need for further research to help health care providers to be effective in improving and reducing variations in outcomes seen in the racial minority population.

\section{Conclusions}

One of the most useful papers on HRQOL in CaP was published by Sanda and associates [11••]. This prospective study of more than 1,200 patients and 625 spouses or partners serves as a landmark paper in the field and should be standard reading for any provider who counsels patients with localized prostate cancer.

QOL is affected in both the short- and long-term for men treated for CaP. QOL issues are important for treating physicians to be familiar with and understand as we strive to provide optimum care to these men and their families along the often lengthy period of the disease trajectory. A considerable impact on the QOL of patients' families is associated with definitive treatment. The mere diagnosis of $\mathrm{CaP}$ and $\mathrm{AS}$ strategies can also negatively affect patients' QOL. Therefore, the patient, spouse or partner, and physician all need to carefully consider, not only the efficacy, but also the consequences of each treatment option, including the potential adverse effects and effect on QOL. Investigations of all new and existing therapies should attempt to gather prospective and objective data on HRQOL. 


\section{References}

Papers of particular interest, published recently, have been highlighted as:

- Of importance

• Of major importance

1. Moul JW, Mouraviev V, Sun L, et al.: Prostate cancer: the new landscape. Curr Opin Urol 2009, 19:154-160.

2. Moore AL, Dimitropoulou R, Lane A, et al.: Population-based prostate-specific antigen testing in the UK leads to a stage migration of prostate cancer. BJU 2009, 104:1592-1598.

3. Jang TL, Yossepowitch O, Bianco F, Scardino PT: Low risk prostate cancer in men under age 65: the case for definitive treatment. Urol Oncol 2007, 25:510-514.

4. Quek M, Penson D: Quality of life instruments in prostate cancer. In Treatment Methods for Early and Advanced Prostate Cancer, edn 1. Edited by Kirby RS, Feneley M. London, Informa Healthcare; 2008:41-44.

5. Meyer KB, Clayton KA: Measurement and analysis of patient reported outcomes. Clin Epidemiol 2008, 473:1-15.

6. van Andel G, Bottomley A, Fossa SD, et al.: An international field study of the EORTC QLQPR25: a questionnaire for assessing the health-related quality of life of patients with prostate cancer. Eur J Cancer 2008, 44:2418-2424.

7. van den Bergh RC, Essink-Bot ML, Roobol MJ, et al.: Anxiety and distress during active surveillance for early prostate cancer. Cancer 2009, 115:3868-3878.

8. Burnet KL, Parker C, Dearnaley D, et al.: Does active surveillance for men with localized prostate cancer carry psychological morbidity? BJU Int 2007, 100:540-543.

9. National Cancer Institute: Observation or radical treatment in patients with prostate cancer. Available at http://clinicaltrials.gov/ct2/show/NCT00499174. Accessed December 2009.

10. Bellizzi KM, Latini DM, Cowan JE, et al.: Fear of recurrence, symptom burden, and healthrelated quality of life in men with prostate cancer. Urology 2008, 72:1269-1273.

11. $\bullet$ Sanda MG, Dunn RL, Michalski J, et al.: Quality of life and satisfaction with outcome among prostate-cancer survivors. N Engl J Med 2008, 358:1250-1261. This article evaluated HRQOL domains among patients and their partners in each prostate cancer treatment modality.

12. GacciM, SimonatoA,Masieri L, et al.: Urinary and sexual outcomes in long-term (5+ years) prostate cancer disease free survivors after radical prostatectomy. Health Qual Life Outcomes 2009, 13:94. 
13. • Chen RC, Clark JA, Talcott JA: Individualizing quality-of-life outcomes reporting: how localized prostate cancer treatments affect patients with different levels of baseline urinary, bowel, and sexual function. J Clin Oncol 2009, 27:3916-3922. This article discusses HRQOL treatment-related complications stratified by baseline categories for sexual, bowel, and urinary function.

14. Coelho RF, Chauhan S, Palmer KJ, et al.: Robotic-assisted radical prostatectomy: a review of current outcomes. BJU Int 2009,104:1428-1435.

15. • Hu JC, Gu X, Lipsitz SR, et al.: Comparative effectiveness of minimally invasive vs open radical prostatectomy. JAMA 2009, 302:1557-1564. Men undergoing minimally invasive versus open prostatectomy RRP experienced more genitourinary complications, incontinence, and erectile dysfunction at the expense of shorter length of stay, and fewer surgical complications.

16. Abel EJ, Masterson TA, Warner JN, et al.: Nerve-sparing prostatectomy and urinary function: a prospective analysis using validated quality-of-life measures. Urology 2009, 73:1336-1340.

17. Nandipati KC, Raina R, Agarwal A, Zippe CD: Nerve-sparing surgery significantly affects long-term continence after radical prostatectomy. Urology 2007, 70:1127-1130.

18. Roeloffzen EMA, Hinnen KA, Battermann JJ, et al.: The impact of acute urinary retention after iodine-125 prostate brachytherapy on health-related quality of life. Int J Radiat Oncol Biol Phys 2009 Nov 23 (Epub ahead of print).

19. Pinkawa M, Fischedick K, Asadpour B, et al.: Health-related quality of life after adjuvant and salvage postoperative radiotherapy for prostate cancer: a prospective analysis. Radiother Oncol 2008, 88:135-139.

20. Sonn GA, Sadetsky N, Presti JC, Litwin MS: Differing perceptions of quality of life in patients with prostate cancer and their doctors. J Urol 2009 182:2296-2302.

Physician ratings of patients' symptoms do not correlate well with patient self assessments of HRQOL.

21. Carlsson S, Aus G, Wessman C, et al.: Anxiety associated with prostate cancer screening with special reference to men with a positive screening test (elevated PSA) - results from a prospective, population-based, randomised study. Eur J Cancer 2007,43:2109-2116.

22. Katz DA, Jarrard DF, McHorney CA, et al.: Health perceptions in patients who undergo screening and workup for prostate cancer. Urology 2007, 69:215-220.

23. Love AW, Scealy M, Bloch S, et al.: Psychosocial adjustment in newly diagnosed prostate cancer. Aust N Z J Psychiatry 2008, 42:423-429. 
24. Reeve BB, Potosky AL, Smith AW, et al.: Impact of cancer on health-related quality of life of older Americans. J Natl Cancer Inst 2009, 101:860-868.

25. Gacci M, Lapini A, Serni S, et al.: Predictors of quality of life after radical treatment for prostate cancer. Urol Int 2008, 80:231-236.

26. Gupta D, Granick J, Grutsch JF, Lis CG: The prognostic association of health-related quality of life scores with survival in breast cancer. Support Care Cancer 2007, 15:387-393.

27. Sadetsky N, Hubbard A, Carroll PR, et al.: Predictive value of serial measurements of quality of life on all-cause mortality in prostate cancer patients: data from CaPSURE (cancer of the prostate strategic urologic research endeavor) database. Qual Life Res 2009, 18:1019-1027.

28. Northouse LL, Mood DW, Montie JE, et al.: Living with prostate cancer: patients' and spouses' psychosocial status and quality of life. J Clin Oncol 2007, 25:4171-4177.

29. Badr H, Taylor CL: Sexual dysfunction and spousal communication in couples coping with prostate cancer. Psychooncology 2009, 18:735-746.

30. Northouse LL, Mood DW, Schafenacker A, et al.: Randomized clinical trial of a family intervention for prostate cancer patients and their spouses. Cancer 2007, 110:2809-2818. Curr Urol Rep (2010) 11:139-146 145.

31. Harden J, Falahee, M, Bickes J, et al.: Factors associated with prostate cancer patients' and their spouses' satisfaction with a family based intervention conclusion. Cancer Nurs 2009, 32:482-492.

32. Jayadevappa R, Johnson JC, Chhatre SE, et al.: Ethnic variation in return to baseline values of patient-reported outcomes in older prostate cancer patients. Cancer 2007, 109:2229-2238.

33. Do YK, Carpenter WR, Spain P, et al.: Race, healthcare access and physician trust among prostate cancer patients. Cancer Causes Control 2010, 21:21-40.

34. Hinz A, Krauss O, Hauss JP, et al.: Anxiety and depression in cancer patients compared with the general population. Eur J Cancer Care 2009 (in press). 


\begin{tabular}{|c|c|}
\hline Treatment Modality & HRQOL Side Effects \\
\hline AS & $\begin{array}{l}\text { Greater level of depression (8) } \\
\text { Higher anxiety score (7) }\end{array}$ \\
\hline $\mathbf{R P}$ & $\begin{array}{l}\text { Greater level of depression }(33) \\
\text { Lower sexual function score }(10,11 \bullet \bullet, 12) \\
\text { Lower urinary function score }(10,11 \bullet \bullet, 12) \\
\text { Lower urinary incontinence score, but improved urinary } \\
\text { irritation or obstruction symptoms }(11 \bullet \bullet)\end{array}$ \\
\hline EBRT & $\begin{array}{l}\text { Greater level of depression }(33) \\
\text { Lower sexual function score, worsened with ADT }(1 \mathbf{1} \bullet \bullet) \\
\text { Lower bowel function score }(11 \bullet \bullet) \\
\text { Lower physical HR-QOL, worsened with ADT }(1 \mathbf{1} \bullet \bullet)\end{array}$ \\
\hline BT & $\begin{array}{l}\text { Greater level of depression }(33) \\
\text { Lower urinary irritation or obstruction scores }(11 \bullet \bullet, 17) \\
\text { Lower bowel function score }(11 \bullet \bullet)\end{array}$ \\
\hline
\end{tabular}

